\title{
Close frequency pairs in Delta Scuti stars
}

\author{
M. Breger and K. M. Bischof \\ Institut für Astronomie der Universität Wien, Türkenschanzstr. 17, 1180 Wien, Austria \\ Received 23 November 2001 / Accepted 22 January 2002

\begin{abstract}
The majority of the well-studied $\delta$ Scuti stars shows frequency pairs in the power spectra with frequency separations less than $0.06 \mathrm{~cd}^{-1}(0.7 \mu \mathrm{Hz})$ as well as amplitude variability. We examine the interpretation in terms of separate excited stellar pulsation modes, single modes with variable amplitudes, and observational problems. The variable-phase technique, which examines the phase jumps near the times of minimum amplitude of an assumed single frequency, is applied to the extensive data of the star BI CMi, which shows some of the most extreme behavior. The following results are found for the 5 features in the power spectrum which could be explained as single modes with variable amplitudes or as double modes: for three features it can be shown that these are indeed pairs of separate pulsation modes beating with each other: at times of minimum amplitude the phase jumps are observed and both the observed amplitude and phase variations can be predicted correctly by assuming two separate modes of nearly equal frequencies. Artifacts caused by observational error, insufficient frequency resolution or variable amplitudes can be ruled out. A fourth pair has a probable origin in two excited modes, while a 5 th case is inconclusive due to long time scales of variability and small amplitudes. The existence of close frequency needs to be taken into account in planning the lengths of earth-based as well as space campaigns so that sufficient frequency resolution is obtained. Possible reasons for the existence of close frequencies in $\delta$ Scuti stars are considered. They include the dense frequency spacing caused by the presence of mixed modes, rotational splitting as well as near-coincidence of the frequencies of modes with different $\ell$ values (the so-called Small Spacing).
\end{abstract}

Key words. stars: variables: $\delta$ Sct - stars: oscillations - stars: individual: BI CMi

\section{Introduction}

The $\delta$ Scuti stars are variable stars situated in the Classical Instability Strip, which pulsate with a large number of simultaneously excited radial and nonradial modes, which makes them well-suited for asteroseismological studies. The amplitudes of the more dominant modes in the typical $\delta$ Scuti star are a few millimag, which is much higher than found in the Sun. It is now possible for ground-based telescopes to detect a large number of simultaneously excited modes with millimag amplitudes in stars other than the Sun. These studies require hundreds of telescope hours at observatories spread around the world.

If it can be established that $\delta$ Scuti stars contain close frequencies of nonradial pulsation as close as $0.05 \mathrm{~cd}^{-1}$, this has important asteroseismological implications. Such close frequencies cannot be explained by first-order rotational splitting since almost all $\delta$ Scuti stars rotate faster than $10 \mathrm{~km} \mathrm{~s}^{-1}$. The existence of extreme splitting asymmetries, which may be produced in rapidly rotating stars (e. g., Goupil et al. 2000), may produce a close doublet in the power spectrum: the observational evidence for such

Send offprint requests to: M. Breger, e-mail: breger@astro.univie.ac.at theoretically predicted behavior is still missing. Similar frequencies are also predicted in the asymptotic case if the two modes belong to different nonradial degrees with $\ell$ values separated by 2 . In fact, the small frequency separations are used as an important asteroseismological tool. Furthermore, it is presently debated whether the large number of closely spaced modes of mixed $\mathrm{p}$ and $\mathrm{g}$ character predicted by theory is indeed found in evolved stars (e.g., see Breger \& Pamyatnykh 2002). Close modes might provide some evidence for such modes.

It therefore appears prudent to examine whether close frequencies of pulsation exist in $\delta$ Scuti stars and if they do, to look at their properties in order to determine their origin.

It is important to emphasize that the presence of double peaks in the power spectrum of a pulsator, or the emergence of a second peak after prewhitening the first peak at a similar frequency, do not necessarily mean that there exist two pulsation modes with close frequencies. A number of different explanations for such a behavior need to be tested. These range from instrumental effects, methods to find the frequencies of pulsation, to other effects intrinsic to the star. Before one can conclude the presence of closely spaced double modes, the possible problems 
associated with the available data sets need to be investigated in detail, in particular:

(i) The problem of frequency resolution: a crucial requirement for the discovery of close frequencies is sufficient frequency resolution. Loumos \& Deeming (1978) derive a frequency resolution of $1.5 / \Delta T$, where $\Delta T$ is the total span of observations. They make the point that the generally used figure of $1 / \Delta T$ is not really correct from a theoretical point of view. Furthermore, data are not equidistantly spaced and longer time gaps can occur. This can decrease the resolution. In an extreme case, the resolution of two months of observing spaced one year apart corresponds to that of a single month. Poor frequency resolution, alone, does not lead to the detection of a double mode. Nevertheless, the combination of poor frequency resolution with one of the problems listed below, can cause severe problems in correctly interpreting the power spectrum.

(ii) The problem of incorrect prewhitening: in the analysis to deduce the multiple excited modes present in photometric data, prewhitening detected frequencies is a common tool to detect further frequencies. Prewhitening with an incorrect frequency value leaves a spurious peak in the power spectrum with a frequency close to the prewhitened frequency, i.e., a doublet is found which does not correspond to two separate modes in the star. Consequently, it is very important to examine every detected frequency doublet whether a single frequency with a better value can explain the doublet.

(iii) Artifacts of amplitude variability of a single mode: amplitude variability on the time scale of years (or even months) is a common feature in $\delta$ Scuti stars (e.g., see Breger 2000a; Arentoft et al. 2001). Some $\delta$ Scuti stars are known to exhibit very strong amplitude variability, e.g., the star $4 \mathrm{CVn}$ with an average annual amplitude variability of $12 \%$ for the different pulsation modes and $40 \%$ over a decade (Breger 2000a). The techniques used to detect the frequencies of pulsation usually rely on constant amplitudes. The amplitude variability shows up in the power spectrum as multiple peaks. Strictly speaking, amplitude variability associated with a single frequency does not lead to a double peak, but to a more complex structure. However, with amplitude variability the exact value of the pulsation frequency can sometimes not be determined, so that the problem of incorrect prewhitening also occurs. In some cases, with amplitude variability only a second peak with sufficient power is noticed, while the other features blend into the noise present in the power spectrum.

(iv) Observational problems: these range from small systematic time errors in the data from one of the multiple observing sites to different effective wavelengths for nominally similar filters at different sites. The latter is caused by the fact that the amplitudes of $\delta$ Scuti stars are extremely wavelength dependent. These problems should be carefully checked for by the observers during data reduction, since at a later stage it is difficult to discover such effects. (v) Effects of aliasing: in a multiple-mode star, aliasing may result in combinations of peaks with incorrect frequencies. Prewhitening such peaks, again, leaves artifacts in the power spectrum, which can be interpreted as double modes. For small-amplitude modes, we regard this effect as one of the most dangerous possibilities. This needs to be examined carefully with statistical programs specializing in the discrimination between various multifrequency solutions, such as PERIOD (Breger 1990) and PERIOD98 (Sperl 1998).

\section{2. $\delta$ Scuti stars with reported close frequencies}

A number of $\delta$ Scuti stars have been reported to have close frequency pairs with separations less than $0.1 \mathrm{c} / \mathrm{d}$. In order to estimate whether this is a common occurrence, we have examined the literature for all stars listed in the $\delta$ Scuti star catalogue (Rodríguez et al. 2000). For most of these stars, very little information is available, so that the question of close frequencies cannot be addressed. Consequently, we selected those stars which were well-studied so that close frequencies (if they exist) could be found. Due to the inhomogeneity of the data, no rigorous, consistent definition is possible. Therefore we selected stars with more than 200 hours of photometric observations, annual observing runs of 20 days or longer, as well as no obvious problems of analysis, measurements, and the distribution of the observations in time. 17 stars could be selected. Of these, seven stars had reported close frequencies. This underestimates the true occurrence because many $\delta$ Scuti stars have very small amplitudes so that only a few modes are observed. If we further restrict the sample to those stars for which more than ten frequencies of pulsation had been found, every one of the seven stars had at least one reported close frequency pair with separations of less than $0.06 \mathrm{c} / \mathrm{d}$. This is surprising, since the frequencies typically cover a wide range between 5 and $15 \mathrm{c} / \mathrm{d}$. Due to inhomogeneity of the data and the small number of wellstudied stars, one should be careful not to overinterpret these numbers. A safe conclusion would be that the majority of well-studied $\delta$ Scuti stars show close frequency pairs.

Table 1 lists the seven stars with reported close frequency pairs with separations of $0.06 \mathrm{c} / \mathrm{d}$ or less.

Let us mention two of these stars: Alvarez et al. (1998) reported two close frequency pairs for the star BW Cnc in the Praesepe clusters. The pairs at 138.7/139.1 and $260.6 / 261.5 \mu \mathrm{Hz}$ have separations of 0.4 and $0.9 \mu \mathrm{Hz}$, respectively. This translates to 0.035 and $0.078 \mathrm{~cd}^{-1}$, respectively. The length of the campaign of $26 \mathrm{~d}$ suggests a frequency resolution of $0.058 \mathrm{~cd}^{-1}$, according to the Deeming \& Loumos criterion so that at least one of the reported doublets has sufficient frequency resolution. Another star is BI CMi, which was studied extensively by the Delta Scuti Network for a number of years so that a frequency separation as small as $0.005 \mathrm{~cd}^{-1}$ could still be resolved.

Some of these studies are quite extensive. Due to the large amounts of data it appears inappropriate to dismiss 
Table 1. $\delta$ Scuti stars with reported close frequencies.

\begin{tabular}{|c|c|c|c|c|c|c|}
\hline Star & $\begin{array}{l}\text { Frequency } \\
\quad\left(\mathrm{cd}^{-1}\right)\end{array}$ & $\begin{array}{l}\text { Frequency } \\
\quad\left(\mathrm{cd}^{-1}\right)\end{array}$ & $\begin{array}{l}\text { Separation } \\
\left(\mathrm{cd}^{-1}\right)\end{array}$ & $\begin{array}{l}\text { Resolution } \\
\quad\left(\mathrm{cd}^{-1}\right)\end{array}$ & Sufficient? & Reference \\
\hline $\mathrm{BI} \mathrm{CMi}$ & $\begin{array}{c}4.783 \\
8.641 \\
10.429 \\
12.327\end{array}$ & $\begin{array}{c}4.818 \\
8.658 \\
10.437 \\
12.350\end{array}$ & $\begin{array}{l}0.035 \\
0.017 \\
0.008 \\
0.023\end{array}$ & $\begin{array}{l}0.003 \\
0.003 \\
0.003 \\
0.003\end{array}$ & $\begin{array}{l}\text { yes } \\
\text { yes } \\
\text { yes } \\
\text { yes }\end{array}$ & Breger et al. (2002) \\
\hline XX Pyx & $\begin{array}{l}27.011 \\
38.065\end{array}$ & $\begin{array}{l}27.102 \\
38.110\end{array}$ & $\begin{array}{l}0.091 \\
0.045\end{array}$ & $\begin{array}{l}0.019 \\
0.019\end{array}$ & $\begin{array}{l}\text { yes } \\
\text { yes }\end{array}$ & Handler et al. (2000) \\
\hline HD 18878 & 11.178 & 11.219 & 0.041 & 0.025 & yes & Mantegazza \& Poretti (1993) \\
\hline $4 \mathrm{CVn}$ & $\begin{array}{l}5.048 \\
6.404 \\
6.680\end{array}$ & $\begin{array}{l}5.134 \\
6.440 \\
6.750\end{array}$ & $\begin{array}{l}0.086 \\
0.036 \\
0.070\end{array}$ & $\begin{array}{l}0.028 \\
0.028 \\
0.028\end{array}$ & $\begin{array}{l}\text { yes } \\
\text { yes } \\
\text { yes }\end{array}$ & Breger et al. (1999) \\
\hline FG Vir & 24.200 & 24.228 & 0.028 & 0.039 & no & Breger et al. (1998) \\
\hline BV Cir & $\begin{array}{l}11.077 \\
12.289\end{array}$ & $\begin{array}{l}11.128 \\
12.381\end{array}$ & $\begin{array}{l}0.051 \\
0.092\end{array}$ & $\begin{array}{l}0.047 \\
0.047\end{array}$ & $\begin{array}{l}\text { yes } \\
\text { yes }\end{array}$ & $\begin{array}{l}\text { Mantegazza et al. (2001) } \\
\text { Kurtz (1981) }\end{array}$ \\
\hline BW Cnc & $\begin{array}{l}11.984 \\
22.516\end{array}$ & $\begin{array}{l}12.018 \\
22.594\end{array}$ & $\begin{array}{l}0.035 \\
0.078\end{array}$ & $\begin{array}{l}0.052 \\
0.052\end{array}$ & $\begin{array}{l}\text { no } \\
\text { yes }\end{array}$ & $\begin{array}{l}\text { Alvarez et al. (1998) } \\
\text { Michel et al. (1999) }\end{array}$ \\
\hline
\end{tabular}

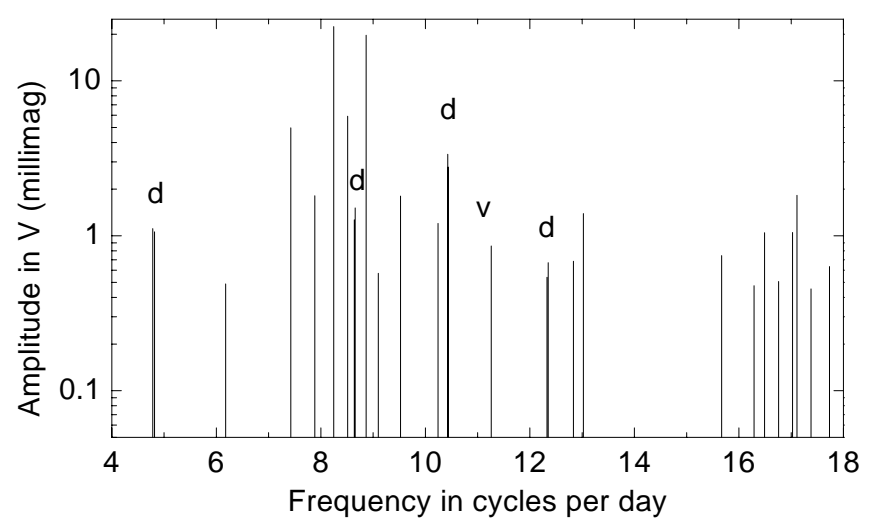

Fig. 1. The pulsation frequency spectrum of BI CMi from $4-18 \mathrm{~cd}^{-1}$. The double peaks in the power spectrum are marked with a "d", while the mode with a highly variable amplitude has been indicated by a "v".

all reports of close frequencies in $\delta$ Scuti stars as due to observational difficulties. It is important to examine a star in close detail to test the different interpretations.

We have chosen the star BI CMi for these detailed examinations. The choice was motivated by the fact that the star has extensive observations available and that we are intimately familiar with the data, since they were obtained by the Delta Scuti Network located in Vienna.

After the pioneering study of BI CMi by Mantegazza \& Poretti (1994), from 1997 to 2000, the Delta Scuti Network obtained more than 1000 hours of photometric data and deduced more than 25 frequencies of pulsation (Breger et al. 2002). The 1998/1999 and 1999/2000 observing seasons covered $125 \mathrm{~d}$ and $109 \mathrm{~d}$, respectively. This provides a frequency resolution of $0.003 \mathrm{~cd}^{-1}$ for the two years. One of the important and puzzling results of the study concerns the presence of five features in the power spectrum which can not be explained as single pulsation modes with constant amplitudes. Their unusual nature modes was discovered when prewhitening the frequency of each mode revealed an additional peak in the power spectrum with a frequency very close to that of the prewhitened mode and/or when different amplitudes were found for different years. We note that the detection of these features is statistically very significant (amplitude signal/noise ratio as high as 6), so that their reality will not be questioned here. The frequency spectrum of BI CMi is shown in Fig. 1, where the location of these features is also shown.

Before the nature of these features is examined in details, one should eliminate the possibility of their origin due to observational uncertainties, complex aliasing effects from other modes or incorrect prewhitening. While such problems can never be eliminated completely, except for feature $5\left(11.26 \mathrm{~cd}^{-1}\right)$ we consider them extremely unlikely as an explanation for the following reason: the data were subdivided into various subgroups based on year and observatory and a large number of new multifrequency solutions with a variety of hypothetical scenarios computed. The result was always the same: two modes with close frequencies or amplitude variability. In the case of feature 5 , we note the existence of a pulsation mode at $10.24 \mathrm{~cd}^{-1}$ with a similar amplitude, so that interaction through $1 \mathrm{~cd}^{-1}$ aliasing presents a potential influence. Again, no obvious alternate explanation could be derived, but for this feature we would like to advise some caution.

Two explanations remain: pairs of close frequencies and amplitude variability. The next section will concentrate on distinguishing between these possibilities.

\section{Close frequency pairs or just amplitude variability?}

In principle, the choice between the hypotheses looks simple: one needs to consider both models and calculate 
Table 2. Parameters for the single- and double mode interpretations.

\begin{tabular}{ccccc}
\hline Feature & $\begin{array}{c}\text { Single-frequency model } \\
\text { Frequency }\end{array}$ & $\begin{array}{c}\text { Double-frequency model } \\
\text { Amplitude in } y \\
\text { (millimag) }\end{array}$ & $\begin{array}{r}\text { Frequency } \\
\left(\mathrm{cd}^{-1}\right)\end{array}$ & $\begin{array}{c}\text { Amplitude in } \\
\text { (millimag) }\end{array}$ \\
\hline 1 & 10.4314 & 0.6 to 6.5 & 10.4289 & 3.6 \\
& & & 10.4365 & 2.7 \\
2 & \multirow{2}{*}{8.6490} & 0.2 to 2.5 & 8.6578 & 1.4 \\
& & & 8.6405 & 1.2 \\
3 & \multirow{2}{*}{4.7826} & 0.3 to 2.7 & 4.7826 & 1.3 \\
& & & 4.8179 & 1.0 \\
4 & \multirow{2}{*}{12.3500} & 0.2 to 1.3 & 12.3500 & 0.8 \\
& & & 12.3268 & 0.5 \\
\hline
\end{tabular}

Note that the observed amplitude range for the single-frequency model does not correspond exactly to the sums and differences of the amplitudes of the double-frequency model since the derived parameters are based on observed data.

optimum fits of the relevant parameters to the data. The models with the lowest residuals between fit and observations would then be chosen. This valid method has one serious drawback: in a multiperiodic star, the two models may lead to only slightly different residuals. Furthermore, the solution with the lowest residuals may not be the correct one due to complex aliasing effects (see above) and the different number of free parameters fitted.

It appears prudent to adopt a more classical, analytical approach. The two hypotheses both lead to a variable amplitude, but to very different phasing behavior: two beating frequencies appear as a single frequency with variable phase. The most extreme case occurs for two frequencies with the same amplitudes: there is a half cycle phase jump at every new cycle of the beat period. Even if the amplitudes are not identical, the phase jump still exists, but is progressively smeared out in time as the amplitudes become more and more unequal. A numerical modeling of the amplitude ratios and phase shifts can be seen in Breger (1981), where the two-frequency model could be ruled out for the cepheid HR 7308.

To distinguish between the two hypotheses, we will examine the amplitude and phase variability for each mode of BI CMi and compare the one- and two-frequency models in the time, amplitude and the time, phase domains. All computations of amplitudes and phases of the mode to be tested for duplicity were carried out simultaneously with all the other frequencies or frequency pairs known for BI CMi as listed in Breger et al. (2002). The simultaneous calculation eliminates any bias caused by spectral leakage from other modes which were assumed to have constant annual amplitudes. Numerical simulations showed, however, that such bias is very minor to start with. The available data have been obtained through the Stromgren $y$ and $v$ filters. We have used both filters to reduce the noise. For the analysis, the $v$ amplitudes were multiplied by an experimentally determined factor of 0.632 to agree with the $y$ amplitudes and any small shifts between the times of maximum for the two filters are ignored. This is a safe procedure because in the test we are looking for phase jumps of up to half a cycle. Furthermore, the results are insensitive to the chosen value for the amplitude ratio because of the similar coverage within each for year for the two colors. The data, which were obtained at different observatories with different detectors and filters, were examined for systematic differences (which might lead to errors in the power spectra). No systematic effects or problems were found.

The test relies mainly on the phase shifts of the assumed single frequency. The results can be represented in different graphical forms based on the uncertainties of the calculated points and the number of measured data points required for each phase range considered. Consequently, we have chosen slightly different graphical forms for demonstrating the results for the different frequencies and to strengthen the conclusions.

Table 2 lists the four best candidates for double modes in BI CMi and shows the relevant parameters for both the two-frequency and single-frequency hypotheses.

\subsection{The mode(s) near $10.43 \mathrm{~cd}^{-1}$}

The pulsation mode near $10.43 \mathrm{~cd}^{-1}$ shows strong amplitude variability on the timescale of about $132 \mathrm{~d}$. To apply the phase test we have assumed the single (best) frequency of $10.43142 \mathrm{~cd}^{-1}$ and computed separate solutions of the amplitudes and phases in 10 to $15 \mathrm{~d}$ intervals. This could be done for all three observing seasons. The separate results were then plotted together utilizing a beat frequency of 132 d. The results are shown in Fig. 2.

We consider the results as surprisingly unambiguous and find:

- The rapid phase change is found. The fact that the phase is almost constant outside the times of the rapid 
switch indicates that we have chosen a correct trial period. The rapid phase change is incompatible with the single frequency/variable amplitude hypothesis and a beating phenomenon of two close periods with similar amplitudes may be involved.

- The fast phase changes occur near the times of minimum amplitude. This is another requirement of the two-frequency hypothesis.

- Both the 1998/1999 and 1999/2000 observing seasons show exactly the same behavior. Apart from confirming our conclusions in favor of the two-frequency hypothesis this result indicates that the amplitudes of the two pulsation modes are not strongly variable on an annual basis.

- The 7 nights of data from 1997 spanning 15 days can provide only one point in Fig. 2. This point is in agreement with the other data and supports the twofrequency model.

- We have computed the best values of the two frequencies, amplitudes and phases by a least-squares fit. In fact, the two-frequency model successfully predicts both the observed amplitudes and phases for all three observing seasons.

We conclude that the model with a single frequency and variable amplitudes can be ruled out from the observations and that the model with two close frequencies provides excellent fits to the data.

\subsection{The mode(s) near $8.65 \mathrm{~cd}^{-1}$}

The successful analysis for the previous (double) mode was repeated for the $8.65 \mathrm{~cd}^{-1}$ mode. Here, the shorter beat cycle of $\sim 56 \mathrm{~d}$ makes the test more difficult than in the previous example, because the time bins for the examination have to be shorter in length and thereby contain fewer points. Figure 3 lists the important phase information for the two extensive 1998/1999 and 1999/2000 observing seasons. The data were plotted on the same diagram with different symbols by adopting an experimentally determined best shift of $335 \mathrm{~d}$ ( 6 beat cycles).

As for the previously examined mode, there exist regular phase jumps of $\sim$ half a cycle. The beat cycle is short enough for each observing season to contain two of these phase discontinuities. These jumps occur at minimum amplitude.

We conclude that the mode near $8.65 \mathrm{~cd}^{-1}$ is actually double with two close frequencies and that the phase jumps are incompatible with the single mode/variable amplitude hypothesis. As was previously also found for the $10.43 \mathrm{~cd}^{-1}$ pair, the amplitudes associated with the two modes are constant or nearly constant on an annual basis.

\subsection{The mode(s) near $4.8 \mathrm{~cd}^{-1}$}

The one or two modes near $4.8 \mathrm{~cd}^{-1}$ are even more difficult to analyze because of the short beat cycle of $28.3 \mathrm{~d}$ and the small amplitudes near $1 \mathrm{mmag}$. Consequently, it was no

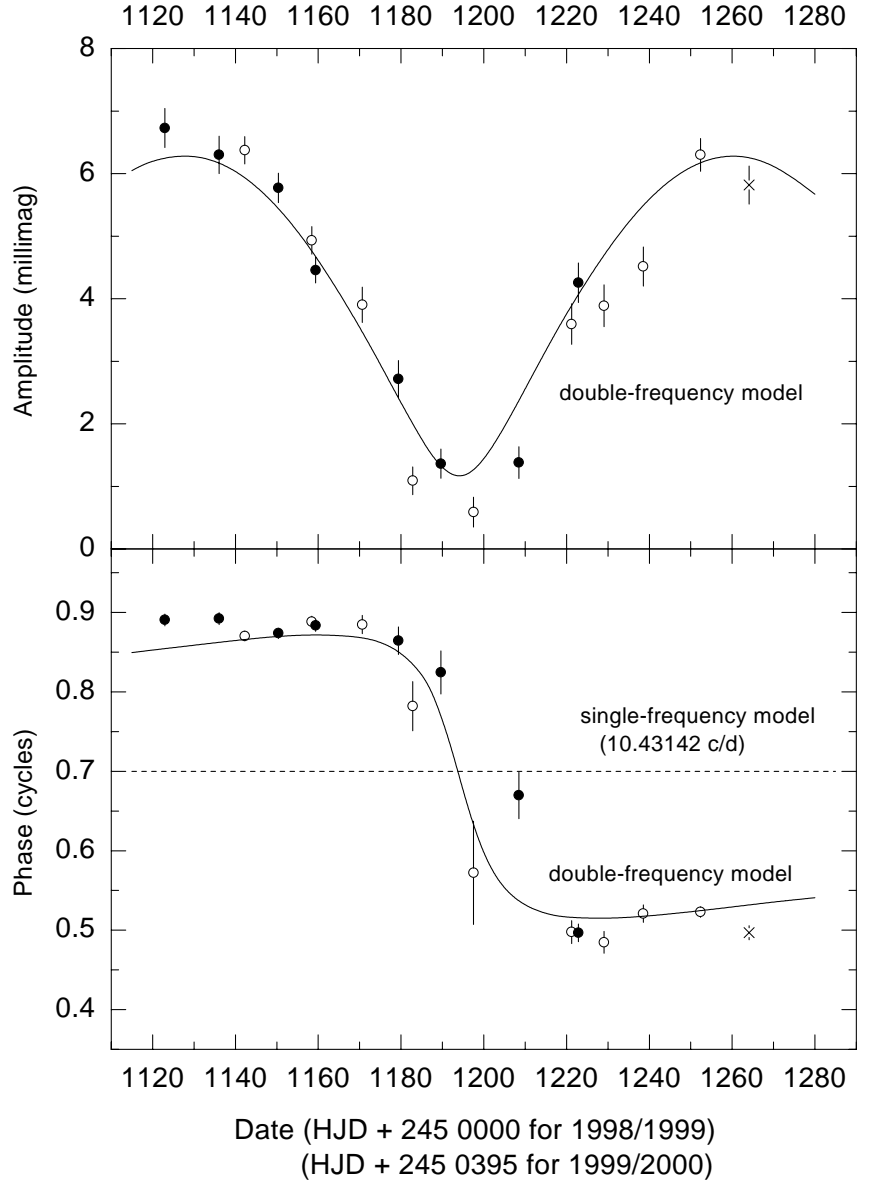

Fig. 2. Amplitude and phase shift diagrams to test whether the particular mode is a single frequency with variable amplitude or composed of two close frequencies with nearly equal amplitudes. Closed circles: 1998/1999 season, open circles: 1999/2000 season, cross: 1997 . The rapid phase change in the middle of the diagram demonstrates the beating phenomenon and the existence of two close frequencies.

longer feasible to examine different beat cycles separately. The 1998/1999 and 1999/2000 data were combined. The short data set from 1997 was omitted in order to avoid potential errors accumulated from the long time base.

To apply the phase test we have assumed the single (best) frequency of $4.7826 \mathrm{~cd}^{-1}$ and computed separate solutions of the amplitudes and phases for each for the 20 phase bins.

The results are shown in Fig. 4. We can see systematic shifts in the phasing of the assumed single frequency. These shifts correspond to the shifts calculated from the two-frequency hypothesis. Because of the different amplitudes associated with the two modes, a sudden phase jump is not expected in this case.

We conclude that in BI CMi there exist two close frequencies near $4.8 \mathrm{~cd}^{-1}$.

\subsection{The mode(s) near $12.35 \mathrm{~cd}^{-1}$}

The Fourier analyses and multiple least squares solutions show two frequencies at 12.3499 and $12.3268 \mathrm{~cd}^{-1}$. 


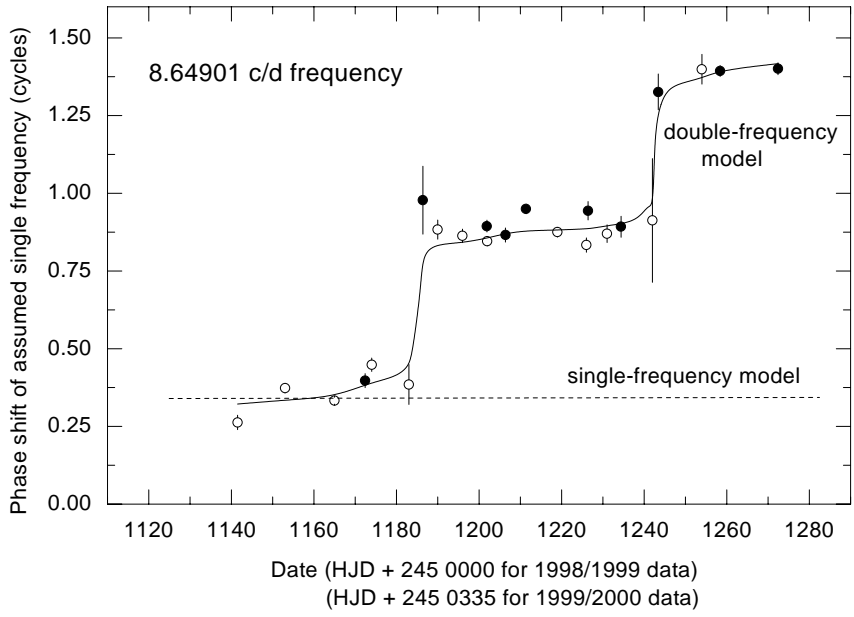

Fig. 3. Phase shift diagrams to test whether the particular mode at $8.65 \mathrm{~cd}^{-1}$ is a single frequency with variable amplitude or composed of two close frequencies with nearly equal amplitudes. Closed circles: 1998/1999 season, open circles: 1999/2000 season. The phase jumps demonstrate the beating phenomenon and the existence of two close frequencies.

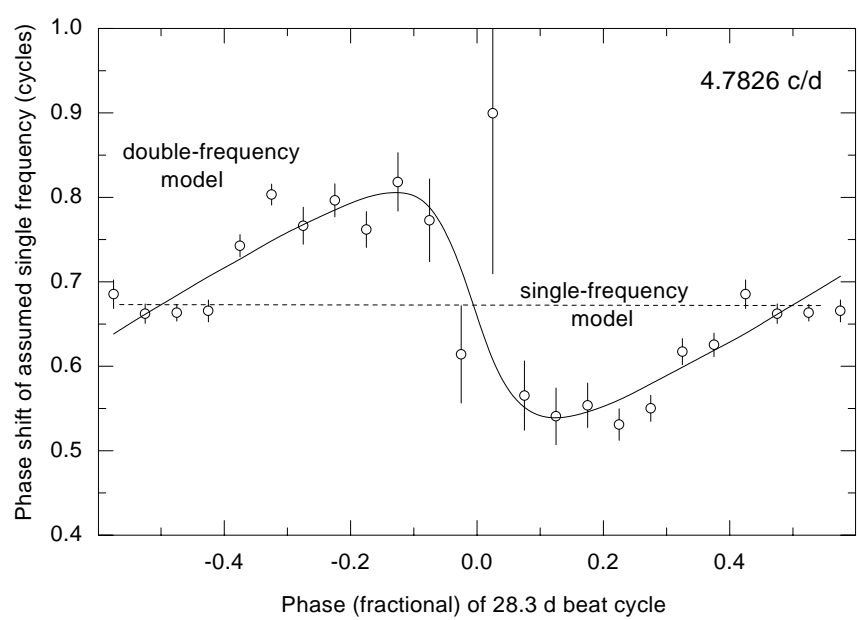

Fig. 4. Phase shift diagram to test whether the particular mode at $4.8 \mathrm{~cd}^{-1}$ is a single frequency with variable amplitude or composed of two close frequencies with unequal amplitudes. The phase shift of an assumed single frequency is calculated for each phase of the beat cycle. The large phasing uncertainty near zero beat phase is caused by the very small amplitude at that phase. The diagram demonstrates the beating phenomenon and the existence of two close frequencies.

This leads to a beat period of $43 \mathrm{~d}$. As before, we have divided the data into 17 bins (each with $\sim 1000$ data points) grouped according to the phasing of the beat period. However, because of the short beat period and the low amplitudes, we were not able find an optimum single frequency to apply the test to. Statistically, the best result always turned out to be one of the frequencies of the frequency pair with a variable amplitude, as would occur by the effect of a neighboring mode or true amplitude variability. We have chosen the frequency with the best residuals, viz., $13.50 \mathrm{~cd}^{-1}$. Figure 5 shows that the phasing test is not conclusive for this case. Both the size of

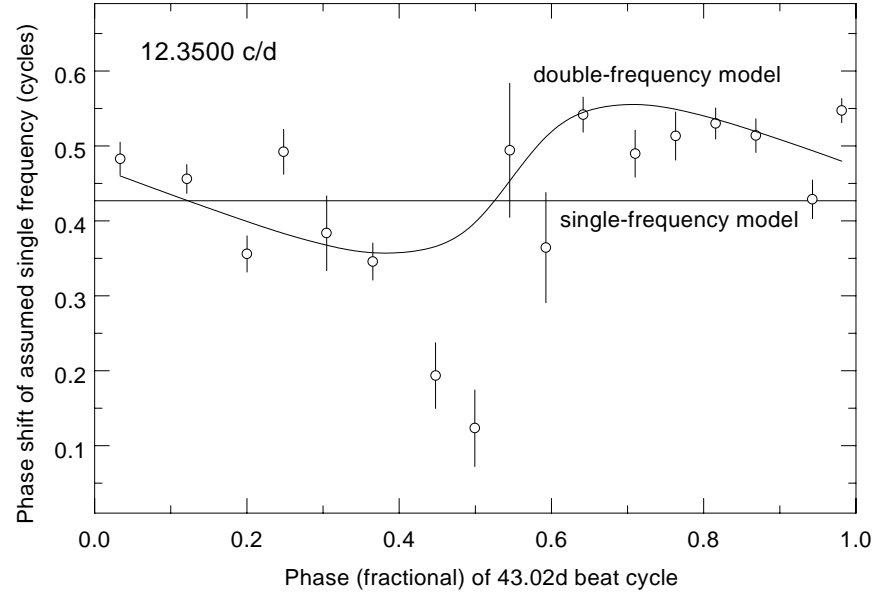

Fig. 5. Phase shift diagram to test whether the particular mode at $12.35 \mathrm{~cd}^{-1}$ is a single frequency with variable amplitude or composed of two close frequencies. The phase shift of an assumed single frequency is calculated for each phase of the beat cycle. The two-frequency hypothesis provides a slightly better fit, but the scatter of the points is too large to convincingly distinguish between the two hypotheses.

the residuals of the multiperiodic solution and the power spectra favor the two-frequency hypothesis over the singlefrequency hypothesis. Nevertheless, a convincing proof is lacking for this frequency pair.

\subsection{The mode(s) near $11.26 \mathrm{~cd}^{-1}$}

The mode at $11.2608 \mathrm{~cd}^{-1}$ shows strong amplitude variability from year to year. During the 1998/99 observing season, covering $120 \mathrm{~d}$, amplitude variability including a minimum was found. On the other hand, no significant variability was found during the 110d of the 1999/2000 observing season. These amplitudes, assuming a single frequency, are shown in Table 3, where the formal uncertainties in the amplitudes and phasing are also shown. As before, the formulae for the computation of these uncertainties assume random errors. The true uncertainties may therefore be somewhat larger.

The amplitude variability indicates a long beat period. Extensive two-frequency modelling shows a beat period between 500 and $600 \mathrm{~d}$, or $\Delta f \sim 0.002 \mathrm{~cd}^{-1}$ between the two (hypothetical) modes. The modelling showed that the two-frequency hypothesis is indeed a strong possibility, but that a unique, reliable solution with two close frequencies could not be found. Including the 1991 data by Mantegazza \& Poretti ( $\sim 2$ mmag amplitude) did not improve the situation because of the long observing gap between 1991 and 1997.

The data in Table 3 shows why the phasing test of the two-frequency hypothesis has to be unreliable: the test relies on the phase change near minimum amplitude. This phase change is detected, viz. $0.12 \pm 0.07$ cycles, but is not statistically significant in the light of the above discussion on the uncertainties. 
Table 3. Amplitude and phase variation of the 11.2608 mode.

\begin{tabular}{cccc}
\hline $\begin{array}{c}\text { Observing } \\
\text { Season }\end{array}$ & $\begin{array}{c}\text { HJD } \\
2460000+\end{array}$ & $\begin{array}{c}y \text { Amplitude } \\
(\mathrm{mmag})\end{array}$ & $\begin{array}{c}\text { Phasing } \\
\text { (cycles) }\end{array}$ \\
\hline 1997 & $2465-2481$ & $2.68 \pm 0.21$ & $0.67 \pm 0.01$ \\
$1998 / 1999$ & $3138-3170$ & $0.64 \pm 0.15$ & $0.61 \pm 0.04$ \\
& $3171-3190$ & $0.34 \pm 0.15$ & $0.53 \pm 0.07$ \\
& $3191-3221$ & $0.99 \pm 0.15$ & $0.66 \pm 0.02$ \\
$1999 / 2000$ & $3222-3257$ & $1.36 \pm 0.15$ & $0.64 \pm 0.02$ \\
& $3514-3549$ & $1.68 \pm 0.12$ & $0.62 \pm 0.01$ \\
& $3550-3583$ & $1.67 \pm 0.12$ & $0.66 \pm 0.01$ \\
& $3584-3624$ & $1.67 \pm 0.12$ & $0.65 \pm 0.01$ \\
\hline
\end{tabular}

We conclude that due to the long beat cycle of the $11.26 \mathrm{~cd}^{-1}$ mode, we cannot distinguish between the variable-amplitude and two-frequency hypotheses.

\section{The strange case of $4 \mathrm{CVn}$}

We have seen that one of the signatures of two close modes is the phase shift of up to half a cycle near minimum amplitude. Such a phase shift has been seen before in another $\delta$ Scuti star: the $7.37 \mathrm{~cd}^{-1}$ mode of $4 \mathrm{CVn}$. This mode exhibits the strongest long-term amplitude variability seen so far. Details can be found in Breger (2000a,b: Figs. 11 and 12). The amplitude dropped from $15 \mathrm{mmag}$ in 1974 to $4 \mathrm{mmag}$ in 1976 and $1 \mathrm{mmag}$ in 1977. In subsequent years, the amplitude has been increasing again. The amplitude variability, by itself, is not unusual: the fascinating change is the phase shift of $0.48 \pm 0.02$ cycles between 1976 and 1977, i.e., at minimum amplitude. The determination of accurate phases became possible when the extensive $1996 / 7$ data of $4 \mathrm{CVn}$ led to accurate frequency values, which also fit the earlier data well.

Although the half cycle shift was suggestive of a double mode, in our previous discussions of $4 \mathrm{CVn}$ we preferred the hypothesis of a single mode which was re-excited at a new epoch. The reason was that numerical simulations of a double mode did not lead to an improvement of the fit to the photometric data relative to that of the singlefrequency variable-amplitude hypothesis. At this stage, we cannot present evidence proving the correctness of either the single-mode or double-mode hypothesis, but note that in the light of the new BI CMi results, the doublemode hypothesis should also be kept in mind for $4 \mathrm{CVn}$. The reason for the less definite conclusions concerning the $7.37 \mathrm{~cd}^{-1}$ feature in $4 \mathrm{CVn}$ is the relative lack of data available for the crucial 1974-1978 time period and the long time gap up to the $1983 / 4$ data. We also note that $4 \mathrm{CVn}$ contains other close frequency pairs such as the 6.404 and $6.440 \mathrm{~cd}^{-1}$ pair. However, if the double-mode hypothesis can be shown to apply to the $7.37 \mathrm{~cd}^{-1}$ feature, then the best two-frequency solution gives a separation of $\Delta f=0.0015 \mathrm{~cd}^{-1}$. This would then be the smallest separation observed in $\delta$ Scuti stars so far.

\section{Possible reasons for close frequency pairs}

There are a number of possible explanations for the close frequency pairs:

(i) Mixed modes: theoretical models predict a very large number of excited nonradial modes in evolved $\delta$ Scuti stars due to the dual nature of these modes ( $p$ modes in the envelope and $g$ modes in the core). This dense spectrum of modes leads to small frequency spacing. The pulsation models of 4 CVn (see Breger \& Pamyatnykh 2002) predict 554 unstable modes with radial orders $\ell=0$ to 2 over a $7 \mathrm{~cd}^{-1}$ range. Since BI CMi is in a similar stage of evolution, the number of modes can be regarded as an estimate for this star as well. The average spacing of $\sim 0.01 \mathrm{~cd}^{-1}$ is of the same order as the separation of the close frequencies found in this paper. If the theoretically predicted modes are indeed unstable, the close frequencies found may find a natural explanation.

We note here that the large number of closely spaced modes is predicted by more than one group using independent codes, e. g., the models for evolved $\delta$ Scuti stars by Guzik, Bradley \& Templeton (2000) show a similar large number of predicted mixed modes.

The problem with this explanation lies in the fact that the large number of theoretically predicted modes of mixed character have not (yet) been discovered. The $\delta$ Scuti stars select only a small number of the possible modes to pulsate with detectable amplitudes and these sometimes show relatively large amplitudes. It is not obvious why the (unknown) mode selection mechanism favors so many close modes (but see below).

(ii) Trapped modes: Breger \& Pamyatnykh (2002) have explored the possibility that the modes with low kinetic energy are preferentially selected since they are excited more easily. These are the modes trapped in the outer envelope and the analogs of pure acoustic modes. In this hypothesis, only the modes with the lowest kinetic energy would be selected out the many possible modes. The effect is very strong for $\ell=1$ and weaker for $\ell=2$ modes. This scenario has been successful in explaining the observed $\ell=1$ frequency spacing in the star $4 \mathrm{CVn}$ and is promising for the $\ell=2$ modes. Again, the arguments could also apply to BI CMi because of its similar evolutionary status.

In spite of the severely reduced number of excited lowdegree modes in the trapped mode hypothesis, two modes with close frequencies are still possible. Calculations of the kinetic energy associated with all the possible modes show that two almost identical modes with neighboring frequencies can have the same low kinetic energy. Both modes might, therefore, be excited.

The average spacing of the mixed-mode hypothesis applies here as well, so that the observed frequency separations can be matched. However, in contrast to the mixed-mode hypothesis, close frequencies would be predicted to occur only for trapped modes, i.e., at a relatively small number of predictable frequencies. We are, at present, engaged in testing this hypothesis with pulsation calculations. 
(iii) Rotational splitting: close frequency pairs can, in principle, be explained by rotational splitting with only two of the split modes visible. A simple calculation independent of the details of stellar models appears to rule out this hypothesis. The transformation from a coordinate system rotating with the star to the observer's coordinate system leads to a frequency separation near $m \Omega$, where $\Omega$ is the frequency of rotation. Since almost all $\delta$ Scuti stars rotate faster than $10 \mathrm{~km} \mathrm{~s}^{-1}$, the predicted frequency splitting is too large. However, this calculation may be too simple because of the extreme splitting asymmetries which can be produced in rapidly rotating stars (e. g., Goupil et al. 2000). Such asymmetries produce rotational splitting which can be very much larger or smaller than that given by the $m \Omega$ term. An observational test of this explanation and subsequent theoretical modelling requires reliable observational identification of the the $m$ values associated with each mode. These are not available.

(iv) The small spacing: two modes with only a small frequency difference are expected if their $\ell$ values differ by 2 , at least in the asymptotic case (e. g., Tassoul 1980). This is shown by

$\nu_{n \ell} \simeq(n+\ell / 2+1 / 4+\alpha) \Delta \nu$,

where $n$ is the radial order and $\ell$ the degree of the pulsation mode, $\Delta \nu$ the (large) spacing between successive orders, and $\alpha$ depends on the phase change at the stellar surface and is, in general, a slowly varying function of frequency. This is related to the so-called Small Spacing, which is expected to be an important asteroseismological tool for $\delta$ Scuti stars (see Christensen-Dalsgaard 2000). The fact that many $\ell=0$ and 2 as well as $\ell=1$ and 3 pairs are separated by only very small frequency differences could provide an explanation for the observed close frequencies. In $\delta$ Scuti stars the pulsations are of lower radial order, so that the asymptotic case does not really apply. Nevertheless, models show that if this explanation for the close modes applies, the observed frequencies would provide an important input into the pulsation models.

An important prediction of this explanation would be that the observed close frequency pairs consist of component modes with $\ell$ values differing by 2 . Because of photometric cancellation effects across the surface, the only pairs that we could expect to observe photometrically would be $(0,2)$ as well as $(1,3)$. This can be checked if mode identifications are available. For the close frequency pair in $\mathrm{BI} \mathrm{CMi}$ with the highest amplitudes, the mode identifications from phase differences (see Breger et al. 2002) suggest $\ell=2$ for $10.429 \mathrm{~cd}^{-1}$ and $\ell=0$ (or maybe 1 ) for $10.437 \mathrm{~cd}^{-1}$. The available mode identifications for the other modes and the observed frequency spacings between the modes (i.e., the $\Delta \nu$ value from Eq. (1)) are at this stage not uniquely determined. Until more data become available, the $\Delta \ell=2$ explanation cannot be excluded.

(v) Mode coupling: frequency shifts can also be produced by nonlinear mode coupling by modes with the same or even different $\ell$ values (see Buchler et al. 1995;
Goupil 2000). These shifts could lead to close frequency pairs. This effect has had very little observational study and additional discussion is beyond the scope of this paper.

\section{Conclusion}

A literature search has shown that close frequency pairs with separations less than $0.06 \mathrm{~cd}^{-1}$ are common among well-studied $\delta$ Scuti stars. One of the best candidates for a more detailed analysis of this phenomenon is the star BI CMi. The variable-phase technique, which examines the phase jumps near the times of minimum amplitude of an assumed single frequency, was applied to test whether theses pairs are caused by separate excited stellar pulsation modes, single modes with variable amplitudes, or observational problems.

It was shown that at least three features are indeed pairs of separate pulsation modes beating with each other: at times of minimum amplitude the predicted phase jumps were observed. Both the observed amplitude and phase variations were predicted correctly by assuming two separate modes of nearly equal frequencies. Artifacts caused by observational error, insufficient frequency resolution or variable amplitudes could be ruled out. A fourth pair has a probable origin in two excited modes, while a 5th case is inconclusive due to long time scales of variability and small amplitudes.

The existence of close frequencies needs to be taken into account in planning the lengths of earth-based as well as space campaigns so that sufficient frequency resolution is obtained.

Acknowledgements. It is a pleasure to thank Alosza A. Pamyatnykh for many interesting discussions. This investigation has been supported by the Austrian Fonds zur Förderung der wissenschaftlichen Forschung, project number P14546PHY.

\section{References}

Alvarez, M., Hernandez, M. M., Michel, E., et al. 1998, A\&A, 340,149

Arentoft, T., Sterken, C., Handler, G., et al. 2001, A\&A, 374, 1056

Breger, M. 1981, ApJ, 249, 666

Breger, M. 1990, Comm. in Asteroseismology (Vienna), 20, 1

Breger, M. 2000a, MNRAS, 313, 129

Breger, M. 2000b, in Delta Scuti and Related Stars, ed. M. Breger, \& M. H. Montgomery, ASP Conf. Ser., 210, 3

Breger, M., \& Pamyatnykh, A. A. 2002, in Radial and Nonradial Pulsations as Probes of Stellar Physics, ed. C. Aerts, T. Bedding, \& J. Christensen-Dalsgaard, ASP Conf. Ser., 259, 388

Breger, M., Zima, W., Handler, G., et al. 1998, A\&A, 331, 271

Breger, M., Handler, G., Garrido, R., et al. 1999, A\&A, 349, 225

Breger, M., Garrido, R., Handler, G., et al. 2002, MNRAS, 329, 531 
Buchler, J. R., Goupil, M. J., \& Serre, T. 1995, A\&A, 296, 405

Christensen-Dalsgaard, J. 2000, in Delta Scuti and Related Stars, ed. M. Breger, \& M. H. Montgomery, ASP Conf. Ser., 210, 201

Goupil, M.-J., Dziembowski, W. A., Pamyatnykh, A. A., \& Talon, S. 2000, in Delta Scuti and Related Stars, ed. M. Breger, \& M. H. Montgomery, ASP Conf. Ser., 210, 267

Guzik, J. A., Bradley, P. A., \& Templeton, M. R. 2000, in Delta Scuti and Related Stars, ed. M. Breger, \& M. H. Montgomery, ASP Conf. Ser., 210, 247

Handler, G., Arentoft, T., Shobbrook, R. R., et al. 2000, MNRAS, 318, 511

Kurtz, D. W. 1981, MNRAS, 196, 53
Loumos, G. L., \& Deeming, T. J. 1978, Astrophys. Space Sci., 56,285

Mantegazza, L., \& Poretti, E. 1993, A\&A, 274, 811

Mantegazza L., \& Poretti E. 1994, A\&A, 281, 66

Mantegazza, L., Poretti, E., \& Zerbi, F. M. 2001, A\&A, 366, 547

Michel, E., Hernandez, M. M., Houdek, G., et al. 1999, A\&A, 342,153

Rodríguez, E., López-González, M. J., \& López de Coca, P. 2000, in Delta Scuti and Related Stars, ed. M. Breger, \& M. H. Montgomery, ASP Conf. Ser., 210, 499

Sperl, M. 1998, Comm. in Asteroseismology (Vienna), 111, 1 Tassoul, M. 1980, ApJS, 43, 469 